\title{
AL-FEC for Improved Mobile Reception of MPEG-2 DVB-T Transport Streams
}

\author{
David Gozálvez, ${ }^{1}$ David Gómez-Barquero, ${ }^{1}$ Thomas Stockhammer, ${ }^{2}$ and Michael Luby ${ }^{2}$ \\ ${ }^{1}$ Universidad Politécnica de Valencia (UPV), Mobile Communications Group, 46022 Valencia, Spain \\ ${ }^{2}$ QUALCOMM Incorporated, Santa Clara, CA 95051, USA
}

Correspondence should be addressed to David Gozálvez, dagoser@iteam.upv.es

Received 28 February 2009; Accepted 16 July 2009

Recommended by Sandro Scalise

\begin{abstract}
We investigate the use of application layer FEC protection in DVB-T (Digital Video Broadcasting-Terrestrial) networks for the provision of mobile services. Mobile reception is characterized by variations of the received signal caused by fast fading and shadowing. DVB-T was originally designed for fixed and portable reception, and generally does not provide enough quality in mobile environments. The link layer protection mechanism MPE-FEC (Multi Protocol Encapsulation-Forward Error Correction) was standardized in DVB-H (Digital Video Broadcasting-Handheld) for the protection of mobile TV services. Although DVB$\mathrm{T}$ itself does not incorporate any link or application layer protection mechanism, AL-FEC (Application layer Forward Error Correction) protection can be introduced in DVB-T in a backwards compatible way. By means of AL-FEC, it is possible to improve the robustness of DVB-T services for the provision of mobile TV. In this paper, we explain the concept of AL-FEC protection in DVB-T and evaluate its performance by means of laboratory measurements and dynamic simulations with shadowing. We study different configurations of AL-FEC and compare its performance with MPE-FEC. In this paper, we discuss some implementation aspects of AL-FEC in real scenarios and propose an implementation based on Raptor codes and hash sequences. We also present results obtained by a first AL-FEC prototype for DVB-T that demonstrates the feasibility of the approach.
\end{abstract}

Copyright (C) 2009 David Gozálvez et al. This is an open access article distributed under the Creative Commons Attribution License, which permits unrestricted use, distribution, and reproduction in any medium, provided the original work is properly cited.

\section{Introduction}

Digital Terrestrial TV (DTT) networks are being deployed worldwide, and it is planned that DTT services completely replace analogue TV in many European countries by latest 2012. DVB-T (Digital Video Broadcasting-Terrestrial) [1] is the European standard of DTT, and has been adopted by many countries all over the world to provide DTT services. DVB-T was designed for fixed and portable reception and generally does not provide enough robustness in mobile environments. Mobile reception is characterized by fluctuations of the received signal due to fast fading and shadowing. These fluctuations cause the loss of portions of information over time, and challenge the reception of DVB-T services in mobile environments. A main reason for this is the short interleaving performed in the physical layer (limited to one OFDM symbol which corresponds approximately to 1 millisecond).
The European digital mobile TV standard called DVB$\mathrm{H}$ (Digital Video Broadcasting-Transmission System for Handheld Terminals) is a technological evolution of DVB$\mathrm{T}$, and was developed specifically for the provision of mobile TV services. DVB-H reutilizes the physical layer of DVB$\mathrm{T}$ and introduces a set of enhancements in the link layer in order to adapt the transmission to mobile reception. These enhancements are aimed to reduce the terminal power consumption and counteract fast fading. A link layer protection mechanism called MPE-FEC (Multi Protocol Encapsulation-Forward Error Correction) [2] increases the robustness in mobile environments whereas a bursty transmission technique referred to as time slicing reduces the power consumption in receivers up to $90 \%$. Simulations for DVB-H have shown that MPE-FEC provides gains up to $9 \mathrm{~dB}$ for mobile users when compared to DVB-T. Furthermore, the maximum Doppler tolerance increases by about $50 \%$ in mobile channels while reutilizing the physical layer of DVB-T [3]. 
AL-FEC (Application Layer Forward Error Correction) has been standardized in DVB-H for file delivery services. The advantage of AL-FEC is that it can spread the protection over large portions of information. AL-FEC takes advantage of the temporal diversity derived from user mobility by the use of extensive time interleaving (up to minutes or even hours), which increases the robustness of the transmitted information against fast fading and especially, against shadowing and signal outages. AL-FEC has been also proposed for DVB-H streaming services in the form of multi-burst protection [4]. Despite excellent performance, the main drawback of this approach is an increase in the channel switching times, which is considered as a crucial parameter in mobile TV usability.

Despite the fact that the physical layer of DVB-H is compatible with DVB-T, DVB-H encapsulates all the audiovisual information in IP (Internet Protocol) datagrams and generally simulcasts the services with lower quality than the MPEG-2 Transport Stream signal of DVB-T. Consequently, DVB-H requires the allocation of specific bandwidth for the transmission of the mobile TV content.

In some studies as well as even deployments, mobile reception of DVB-T has been verified. In order to enable mobile reception of current DVB-T services, antenna diversity techniques have been proposed. In [5], it is shown that the reception by means of two antennas results in a similar performance to MPE-FEC. However, for handsetbased reception at UHF frequencies, multiple receiving antennas are generally impractical as the correlation distance of the antennas is far beyond the dimensions of typical handsets.

In this paper, we study the use of AL-FEC protection in DVB-T in order to increase the robustness of the transmitted information and to achieve reception in mobile channels. Specifically, we introduce mechanisms that allow the transmission of the additional FEC data needed for error correction (similar to the MPE-FEC in DVB-H) in a backward compatible way, that is, in such a way that the legacy DVB-T receivers are not impacted by this additional FEC. We also evaluate the mobile performance of AL-FEC in DVB-T and provide direct comparisons with MPE-FEC. Although different codes may be used in order to provide AL-FEC protection for DVB-T services, in this paper we consider the use of Raptor codes [6], as they are efficient and lightweight in terms of decoding complexity. This permits the FEC decoding to be done in generic software processors even on low-complexity devices and therefore does not require any upgrades in hardware. The requirements in terms of complexity and memory on mobile terminals are almost negligible as has been shown in the context of 3 GPP MBMS or other mobile TV standards that integrate Raptor codes.

The rest of the paper is organized as follows. In Section 2, we discuss the typical characteristics of mobile reception and the impairments of the mobile channel. In Section 3 we review the transmission of content in DVB-T networks and detail the implementation of AL-FEC in DVB-T. Section 4 is dedicated to the description of the simulation scenario and then, in Section 5, we present selected illustrative simulation results. In Section 6, we also address the issues of the practical implementation of AL-FEC in DVB-T. Section 7 provides considerations on DVB-T network planning for the provision of mobile TV services. Some concluding remarks are provided in Section 8.

\section{Mobile Reception of DVB-T}

Mobile channels are characterized by rapid variations of the received signal over time referred to as fast fading. Fast fading is caused by the Doppler shift of multiple propagation paths, which originate from the movement of the receiver with respect to the transmitter [7]. Fast fading results in the corruption of small portions of the data stream in a bursty manner due to ICI (Inter Carrier Interference). Higher velocities involve higher values of Doppler shift and thus, a greater degradation of the received information. If the user velocity is too high, the Doppler shift may increase above the values supported by the physical layer, corrupting great portions of information. The $\mathrm{C} / \mathrm{N}$ (Carrier to Noise) sensitivity required for the proper reception of DVB-T tends to increase proportionally with the Doppler shift until a maximum Doppler value, from which reception is no longer possible. The protection applied by the physical layer of DVB-T extends only to the duration of one OFDM symbol (approximately 1 millisecond), and it is not capable to cope with the error bursts resulting from fast fading.

DVB-H reutilizes the physical layer of DVB-T, but integrates MPE-FEC at the link layer to repair the errors caused by mobile reception. MPE-FEC is an intra burst mechanism for which the protection is performed on a per burst basis [2]. MPE-FEC protection spreads over the duration of one burst $(0.1-0.4$ seconds) and is capable to counteract the effects of fast fading. By means of MPEFEC it is possible to achieve a performance that is almost independent of the Doppler and in addition, the maximum Doppler supported by the system can be increased. In order to cope with mobile reception impairments in DVBT systems, antenna diversity techniques have been proposed. In [5] it is shown that by means of 2 receiving antennas, it is possible to achieve a similar performance to MPE-FEC against fast fading.

The received signal in mobile channels is also characterized by slow variations known as shadowing. Shadowing results from the presence of large obstacles, such as buildings or hills that may block the line-of-sight between the receiver and the transmitter. Shadowing is generally modelled as a log-normal distributed variation of the received signal over the area of coverage [7]. When a user is moving in the presence of shadowing, the received signal may experience outages that corrupt longer periods of the data stream. Signal outages can be corrected if the protection of the physical or upper layers is spread over time. Such link layer protection has, for example, been standardized in $[8,9]$ for its use in DVB systems.

\section{AL-FEC Protection of DVB-T Services}

3.1. System Architecture. For the integration of AL-FEC into DVB-T, the architecture according to Figure 1 is 


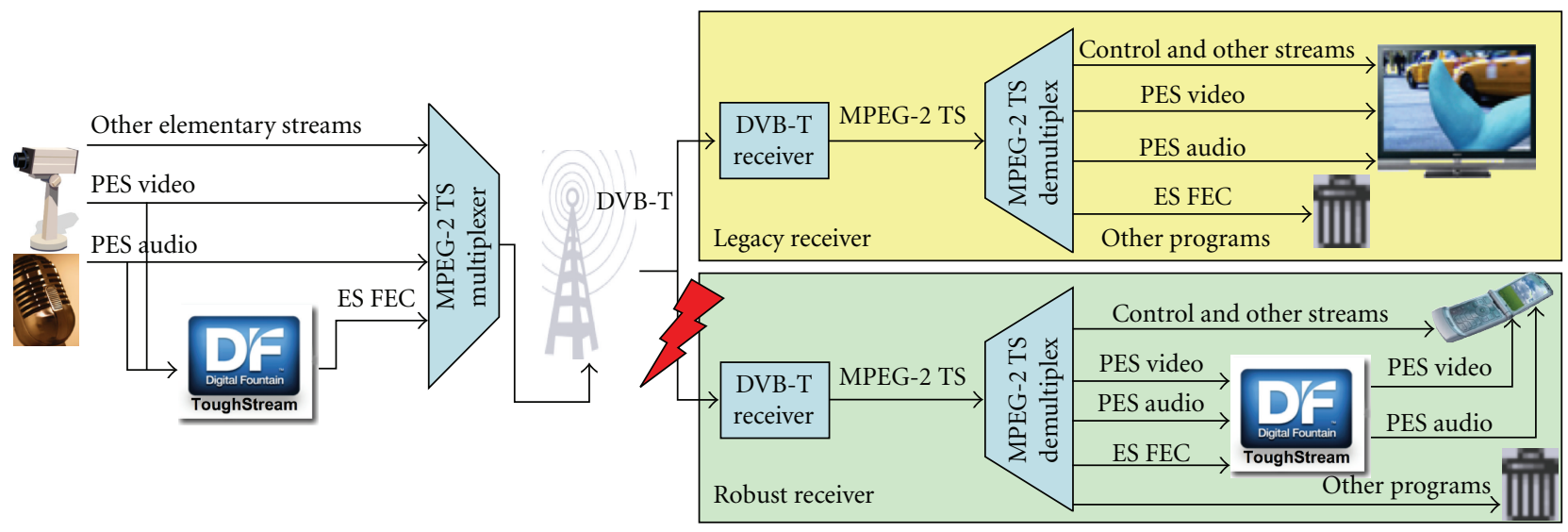

Figure 1: Proposed system architecture.

proposed. The elementary streams of the program to be protected are also processed by an FEC encoder at the transmitter. This entity generates an FEC elementary stream that is multiplexed with the MPEG-2 Transport Stream (TS) and distributed over DVB-T. Legacy receivers receive the MPEG-2 TS and should drop the FEC elementary stream. A robust receiver will make use of the FEC elementary stream to retrieve lost audio and video data, such that the original signal can be reconstructed. The FEC data needs to be multiplexed such that legacy receivers can drop it without impacting their operations. Furthermore, the robust receivers should be able to reconstruct as much data as possible by the use of the FEC elementary stream.

The FEC stream generated by the FEC encoder consumes part of the bit rate capacity at the physical layer and thus, the number of services carried per MPEG-2 TS may have to be reduced in order to accommodate the FEC data. The main difference of the proposed approach with respect to DVB-H lies in the fact that the same multimedia content transmitted to fixed receivers is protected for its use by mobile users. Therefore, no additional content is needed for the transmission of mobile services and only the capacity required for carrying the FEC data must be taken into account to support mobile reception.

Based on this short system description and the presented requirements we will now further detail the different components of the proposed system, in particular the MPEG2 Transport Stream protocol as well as the Forward Error Correction scheme and methods.

3.2. MPEG-2 Transport Stream. In DVB-T all the content is multiplexed in an MPEG-2 TS and transmitted as a sequence of TS packets [10]. Each TS packet carries a header of 4 bytes and a payload of 184 bytes. The MPEG-2 TS contains all the data from the services multiplexed in the MPEG-2 TS along with signalling information, which is carried in the form of PSI/SI (Program Specific Information/Service Information) tables. Generally, several services (TV programs, radio programs, data channels ...) are multiplexed in one MPEG-2 TS. The video, audio and data of each service in the MPEG-2 TS is referred to as Elementary Stream (ES). The header of each TS packet contains a 13-bit Packet IDentifier (PID) that uniquely identifies the ES that is carried inside the TS packet. The header of the TS packets also contains a Transport Error Indicator (TEI) bit and a Continuity Counter (CC) field that can be used for the detection of erroneous and missing packets.

Each ES is assigned a unique PID value inside the MPEG2 TS. The associations between ESs and PID values are transmitted in the PSI/SI tables. DVB-T receivers parse the PSI/SI tables in order to identify the PID values of the ESs corresponding to the desired service. Then, the TS packets carrying the video, audio or data information of the service are demultiplexed by looking at the PID value of every TS packet.

3.3. Forward Error Correction. FEC mechanisms are designed to cope with the loss of information by transmitting additional FEC data. Erasure codes are often used in FEC mechanisms as they can regenerate lost portions of information transmitted over an erasure channel. In order to do so, the information to be protected is partitioned into source blocks, each of them constituted by $k$ different source symbols. An erasure encoder is used in the transmitter to encode the source blocks and generate a total amount of $n$ symbols per source block where $n>k$. If a systematic code is used, the original $k$ source symbols are among the total $n$ symbols generated by the encoding algorithm. The $k$ original source symbols are transmitted along with the $n-k$ repair symbols. Assuming an erasure channel, some of the $n$ transmitted symbols are erased and are not available in the receiver. An erasure decoder is capable to recover the erased symbols if a sufficient number of source and repair symbols is received. An ideal erasure code is capable of recovering the $k$ original source symbols if at least any $k$ symbols among the $n$ transmitted symbols are received. A practical low-complexity erasure code generally requires a small additional number of symbols in order to recover all original transmitted source symbols.

The protection provided by FEC mechanisms depends on the code rate and the protection period. The code rate is the proportion of source data with respect to the 


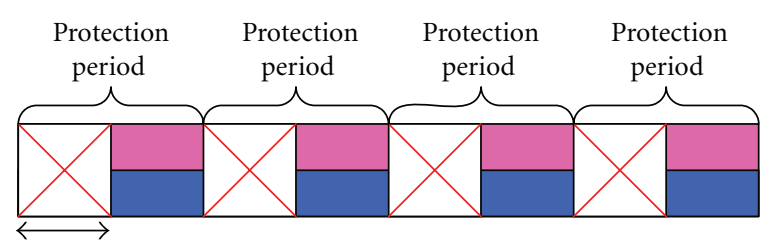

Reparable error burst

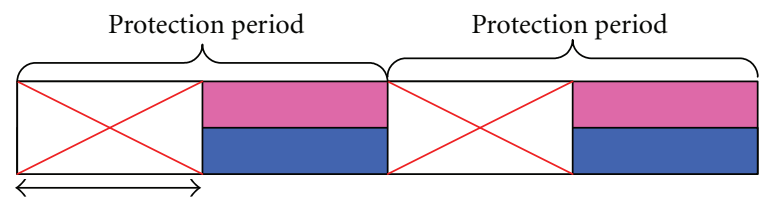

Reparable error burst

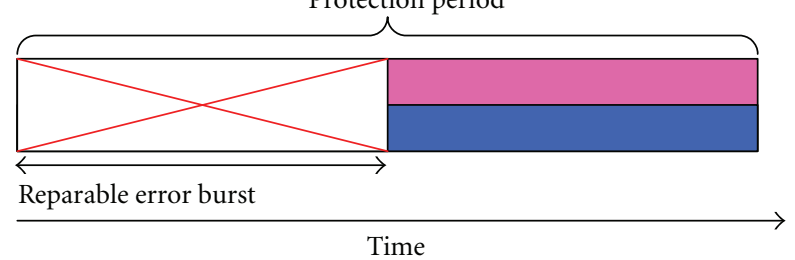

Service data

Parity data

$\bigotimes$ Lost data

FIgURE 2: Example of the impact that the protection period holds on the error correction capabilities of FEC mechanisms.

total amount of information transmitted, accounting both source and repair data. The protection period refers to the duration of the information encoded in each source block. Figure 2 illustrates the effect of the protection period in FEC protection mechanisms. The code rate is assumed to be $1 / 2$ and thus, it is possible to recover the service data by receiving at least half of the total transmitted data. The figure shows the burst correction capabilities for three different protection periods. The lost portions of data illustrated in the figure represent the maximum error burst duration that each protection period is capable of repairing. As can be seen in the figure, longer protection periods operate with greater portions of information and can recover from longer error bursts.

The protection period has an impact not only in the protection provided by the FEC mechanism but also in the network latency and, most importantly, in the receiver latency and channel switching time. The network latency can be defined as the amount of time that passes from the moment the information enters the transmitter till the moment is delivered to the media decoders in the receiver. The channel switching time refers to the amount of time between the instant when the user switches to a new channel and the instant when the new content is displayed to the user. Although network latency is not essential for the majority of services, the channel switching time is considered as a crucial criterion in mobile TV user experience, and must not be increased beyond certain values. There is a trade off between the level of protection that can be offered in mobile reception and the channel switching time that the user experiences as disturbing. Increasing the protection period has also an impact on the memory requirements in the receiver, as at least the size of the source data contained in one protection period must be stored in order to perform the decoding.

3.4. Application Layer FEC. The main idea of AL-FEC in DVB-T is to incorporate FEC protection by making use of erasure codes in a backward compatible way. In order to achieve this, the video and audio ESs must not be altered. Apart from the repair symbols, some additional information such as Source FEC Payload Identifier (ID) is necessary for the decoding process and must be passed to the receiver. For the purpose of connecting source and repair data, hash sequences of the source symbols are sent along with the repair symbols to provide this Source FEC Payload ID. By doing so, the source data is unmodified but the advanced receiver can still retrieve the necessary information by producing the same hash sequences. Moreover, the FEC data, including both the repair symbols and the hash sequences, must be encapsulated in a manner that ensures that legacy DVB-T receivers drop the TS packets carrying the FEC data without altering its proper operation. The MPEG-2 TS specification allows AL-FEC to be incorporated into the protocol stack of DVB-T in a transparent manner above the TS layer. The repair packets can be multiplexed into the MPEG-2 TS as another ES associated to the service, and will be discarded by the DVB-T receivers that do not incorporate AL-FEC. This is accomplished by assigning a specific new PID to the FEC elementary stream that is not recognized by the legacy receiver. By means of the TEI and the continuity counter fields in the TS packet header it is possible to detect erroneous or missing TS packets. As the source and repair packets are encapsulated in TS packets, the erasure of TS packets results in a symbol erasure channel. A source or repair symbol is considered erased if at least one of the TS packets carrying information of that symbol is lost. Longer source and repair packets are usually fragmented and encapsulated into multiple TS packets. As one erroneous or lost TS packet is sufficient to erase the entire source or repair packet, they tend to achieve a lower performance, especially in the presence of uncorrelated MPEG-2 TS packet errors.

In this paper we consider the use of Raptor codes [6] for AL-FEC in DVB-T services. They have been previously standardized in DVB systems for the provision of link layer FEC protection $[8,9]$. Raptor codes are a computationally efficient implementation of fountain codes that achieve very close to ideal performance. Fountain codes are a class of erasure codes that can generate a very large amount of FEC data from a given source block and thus are considered as rate less (i.e., any amount of FEC data can be delivered for any source block size or protection period). Raptor decoding can be implemented in complexity and memory constrained receivers such as handset receivers without the need of dedicated hardware due to its low computational complexity and its efficient memory management.

In Figure 3 we compare the operation of MPE-FEC and AL-FEC. Because of the time slicing performed in DVB-H, the protection period achieved by MPE-FEC is limited to the 


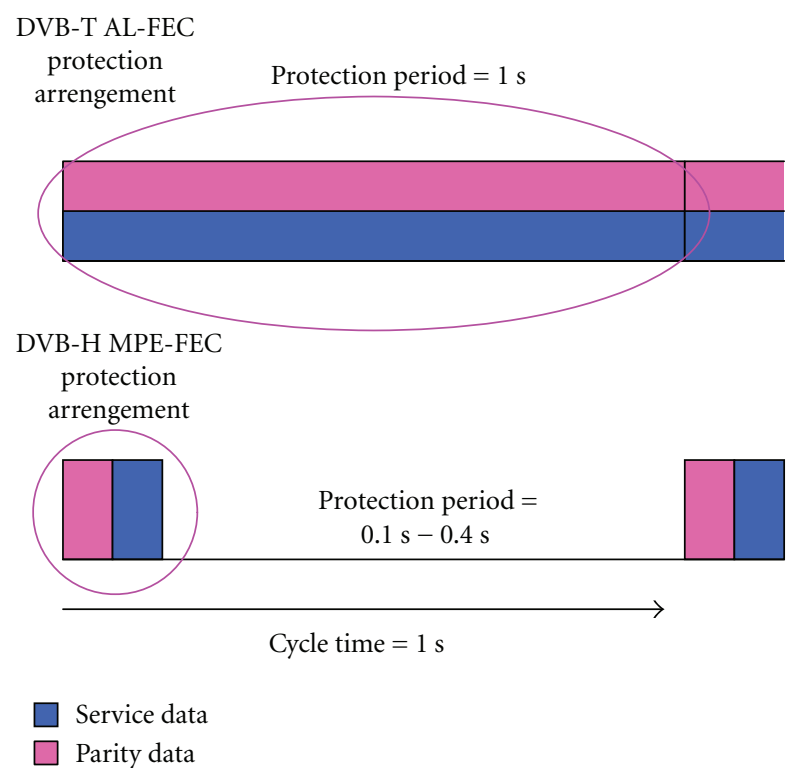

Figure 3: Comparison between the protection arrangement of MPE-FEC in DVB-H and AL-FEC in DVB-T.

burst duration of around 0.1-0.4 seconds. In DVB-H, when a new channel is selected by the user, the receiver must wait until the reception of the first burst of the service. Assuming no other delays, the channel switching time is equal to half the cycle time (i.e., period of time between bursts) on average and as high as one cycle time in the worst-case scenario. On the other hand, DVB-T does not perform time slicing, and the services are transmitted continuously over time. The protection period in AL-FEC can be configured up to 10 seconds and even more, and is only restricted by memory and channel switching time constraints. Although DVB-T does not perform time slicing and thus, it is not possible to achieve power saving, the development of more durable batteries has reduced the power consumption issues in handheld terminals. Furthermore, for a significant portion of mobile TV receivers such as netbooks or in-car receivers, battery lifetime is of less relevance.

Another limitation of MPE-FEC that can be overcome by AL-FEC is the dependency between code rate and protection period. Due to the nature of Reed Solomon encoding, in order to achieve different code rates other than the mother code rate $(3 / 4)$, it is necessary to perform a padding/puncturing mechanism that shortens the burst duration. This is especially critical for code rates $2 / 3$ and $1 / 2$, for which the burst duration is, respectively, a $25 \%$ and a $50 \%$ shorter than in the case of code rate $3 / 4$. On the contrary, the flexibility of Raptor allows the proposed Al-FEC implementation to deliver virtually any code rate for a given protection period.

\section{Performance Evaluation}

The simulations have been performed assuming a DVB$\mathrm{T}$ physical layer configuration of FFT $8 \mathrm{~K}$, guard interval $1 / 4$, nonhierarchical modulation 16QAM, and code rate
$1 / 2$. The transmission mode employed gives a total bit rate of approximately $9.95 \mathrm{Mbps}$. For the evaluation of ALFEC in DVB-T services, a service of $2.5 \mathrm{Mbps}$ has been protected by an ideal AL-FEC implementation. The size of the source and repair symbol is configured to 184 bytes, which corresponds to the payload of one TS packet and therefore each symbol is directly be mapped to one MPEG2 TS packet. Initially, we have considered that no additional overhead (e.g., hash sequences) is required to perform the decoding, so all the FEC data is dedicated to the carriage of repair symbols. These assumptions correspond to an upper limit on the performance, but the expected deviation from real implementations is marginal as we will discuss later. We have also assumed an ideal erasure code for the encoding/decoding algorithm, so the source blocks can be decoded successfully without any additional FEC symbol overhead.

For the MPE-FEC evaluation, we have assumed a DVB$\mathrm{H}$ service with a cycle time of 2 seconds where all the transmitted IP datagrams have a constant size of 512 bytes. The number of rows of the MPE-FEC frame has been configured to 512, and thus, each IP datagram fits exactly in one column of the MPE-FEC frame. The amount of columns of IP information and FEC data were configured according to each particular code rate.

The mobile performance has been evaluated by means of TU6 laboratory measurements. The laboratory measurement setup consists of a DVB-T modulator, a signal generator in charge of emulating the TU6 channel model and a DVB-T measurement system capable of recording the error data at the TS layer. By recording the error data at the TS layer it is possible to emulate the performance of FEC mechanisms in upper layers. The measurements were obtained in a range of CNR values from 0 to $30 \mathrm{~dB}$ and in a range of Doppler values from 5 to $80 \mathrm{~Hz}$.

In order to study the influence of the shadowing, we have assumed a user moving at constant velocity across a log-normal CNR map defined by its standard deviation and correlation distance. The user velocity is given by the values of Doppler and frequency carrier configured in the simulations. The shadowing model outputs instantaneous CNR values that correspond to 100 millisecond time intervals. The instantaneous CNR values along with the Doppler determine the measured error data in each one of the $100 \mathrm{~ms}$ time intervals that will be passed to the link layer.

We have defined two different scenarios: a high diversity scenario with a correlation distance of 20 meters and a Doppler frequency of $80 \mathrm{~Hz}$ (which correspond to $144 \mathrm{~km} / \mathrm{h}$ at $600 \mathrm{MHz}$ ), and a low diversity scenario with a correlation distance of 100 meters and a Doppler frequency of $10 \mathrm{~Hz}$ (which correspond to $18 \mathrm{~km} / \mathrm{h}$ at $600 \mathrm{MHz}$ ). In both cases the standard deviation has been set to $5.5 \mathrm{~dB}$ which is the usual value employed in mobile TV planning for outdoor reception. The results have been averaged over 100 seeds of shadowing.

We have employed the PER (Packet Error Ratio) in order to measure the performance of both MPE-FEC and AL-FEC. The PER is defined as the percentage of packets in which there is at least one error. In the case of MPE-FEC, a packet 


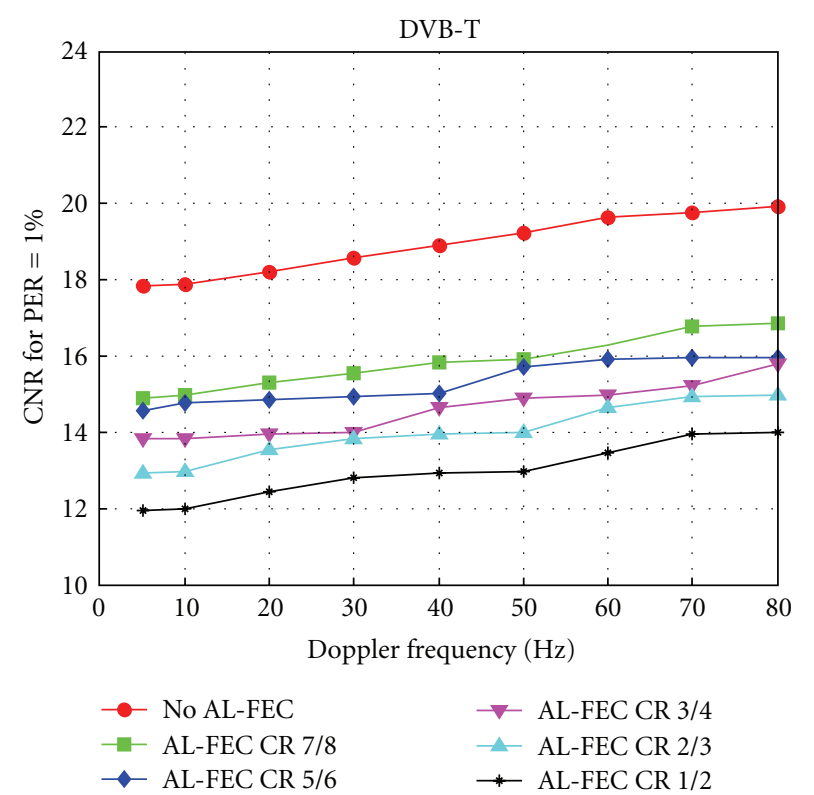

(a)

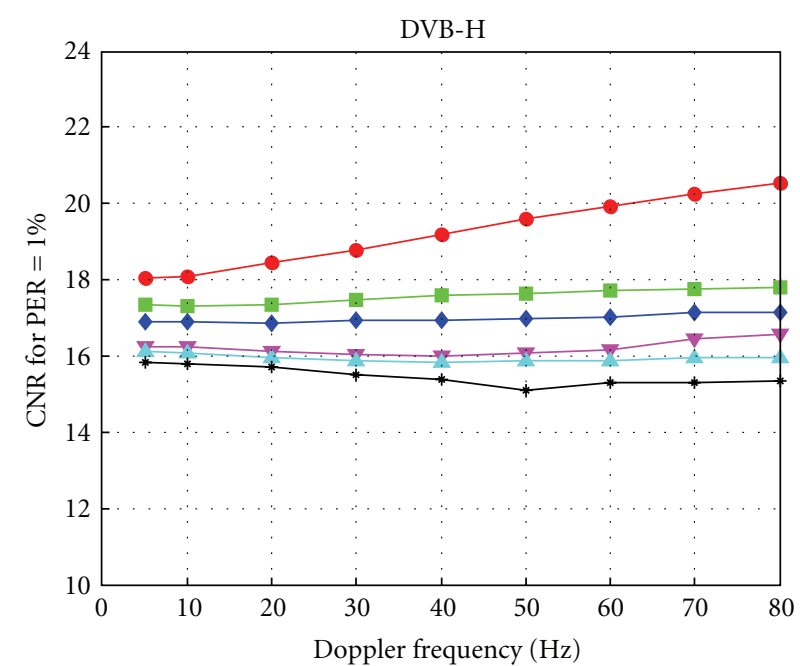

$\longrightarrow$ No MPEFEC
$\rightarrow$ MPEFEC CR 7/8
$\rightarrow$ MPEFEC CR 5/6

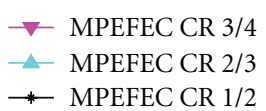

(b)

FIgure 4: Mobile Performance of DVB-T (a) and DVB-H (b) services with AL-FEC in a TU6 channel.

corresponds to a MPE section (in which an IP datagram is encapsulated), whereas in the case of AL-FEC a packet corresponds to a source packet. Although this is not a oneto-one comparison it is a reasonable way to compare the performance of both mechanisms. We have considered a PER value of $1 \%$ as QoS criterion, which is more demanding than the MFER (MPE-FEC Frame Error Ratio) value of 5\% generally employed in DVB-H evaluations.

\section{Results and Discussions}

5.1. Mobile Performance in the Presence of Fast Fading. First, we compare the mobile performance of DVB-T and DVB$\mathrm{H}$ in Figure 4. AL-FEC has been configured to a protection period of 1 second in order to be comparable to the DVB-H service (cycle time equal to 2 seconds) in terms of channel switching time.

As can be seen in the figures, if no link or application layer protection is provided in DVB-H and DVB-T, both systems achieve a very similar performance. DVB-T and DVB-H employ the same physical layer, and the performance difference is due to the source packet size ( 528 bytes in the case of MPE-FEC and 184 bytes in the case of AL-FEC). As it is shown in the figures, the performance degradation due to the packet size is more severe for high Doppler values.

When MPE-FEC and AL-FEC are enabled, the performance of DVB-H and DVB-T increases considerably, achieving important gains in terms of CNR threshold. The gain obtained by MPE-FEC for the lowest code rate is up to $2 \mathrm{~dB}$ in the lower range of Doppler and up to $6 \mathrm{~dB}$ in the higher range. The performance difference between the lowest and highest code rates is around $2 \mathrm{~dB}$. These values correspond quite well with the results presented in [3], which validates the methodology employed in this paper.

The gain obtained by AL-FEC is up to $6 \mathrm{~dB}$ in the entire range of Doppler. This represents an additional gain of $4 \mathrm{~dB}$ with respect to MPE-FEC in the case of low Doppler. This gain is originated by the longer effective protection period of AL-FEC. Despite the fact that MPE-FEC is configured with a cycle time of 2 seconds, the effective protection period is equal to the burst duration. With the configuration employed in the DVB-H service, the burst duration is approximately of $100 \mathrm{~ms}$ for the code rates $7 / 8,5 / 6$ and $3 / 4$, of $80 \mathrm{~ms}$ for the code rate $2 / 3$, and of $50 \mathrm{~ms}$ for the code rate $1 / 2$. On the contrary, AL-FEC is configured with a protection period of 1 second that is independent of the code rate. The errors caused by low Doppler shifts tend to be more correlated over time, and longer protection periods can cope more effectively with this kind of error distributions.

Figures 5 makes a direct comparison between MPE-FEC and AL-FEC with different values of protection period. As we have seen, the effective protection period achieved by MPEFEC in the simulations is between 50 and $100 \mathrm{~ms}$. In MPEFEC, lower code rates operate with higher percentages of FEC data but involve shorter protection periods. As expected, in the case of code rates 7/8,5/6, and 3/4, MPE-FEC obtains results that match those of AL-FEC with a protection period of 0.1 seconds. In the case of code rates $2 / 3$ and $1 / 2$, the performance of MPE-FEC degrades slightly with respect to AL-FEC due to the shorter burst duration. It is also remarkable the fact that with $10 \mathrm{~Hz}$ of Doppler, 1 second of protection period losses less than $1 \mathrm{~dB}$ with respect to 5 and 10 seconds.

Figure 5 shows again how higher values of Doppler degrade more severely the performance of MPE-FEC due 


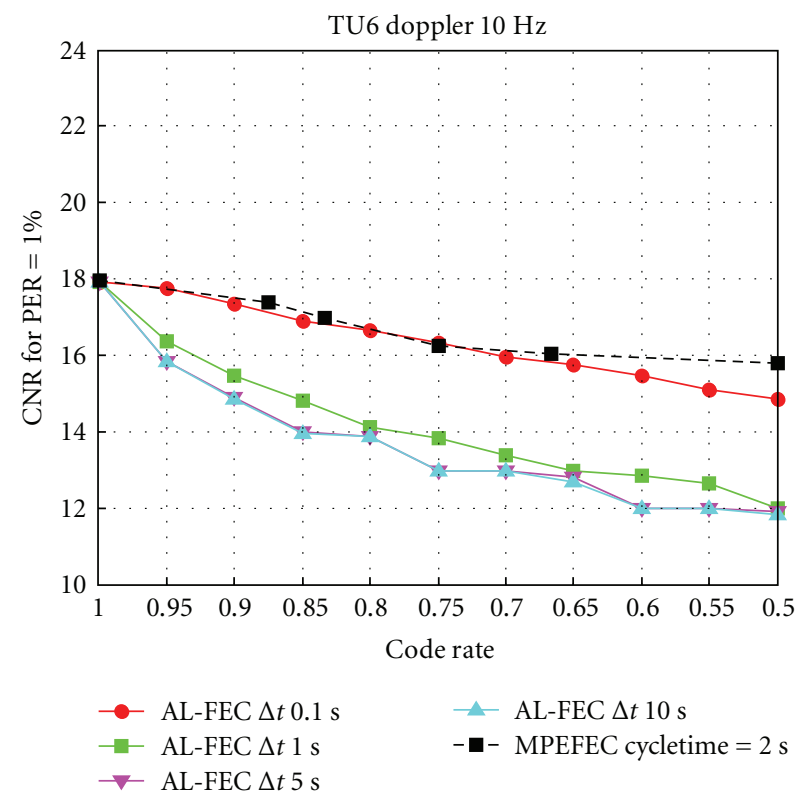

(a)

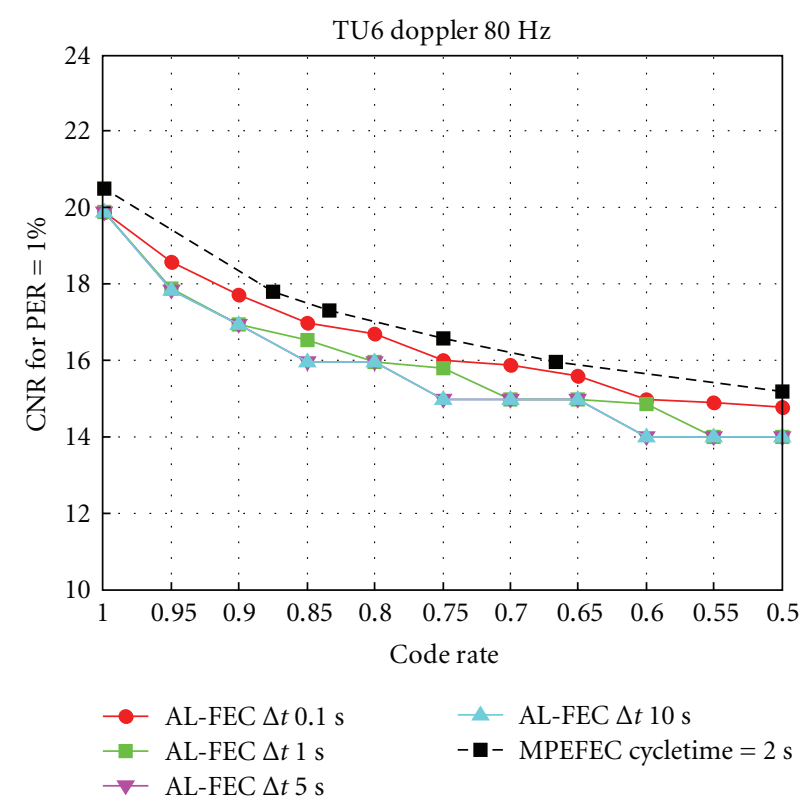

(b)

FIgURE 5: Performance of AL-FEC in a TU6 channel configured with $10 \mathrm{~Hz}$ (a) and $80 \mathrm{~Hz}$ (b) of Doppler.
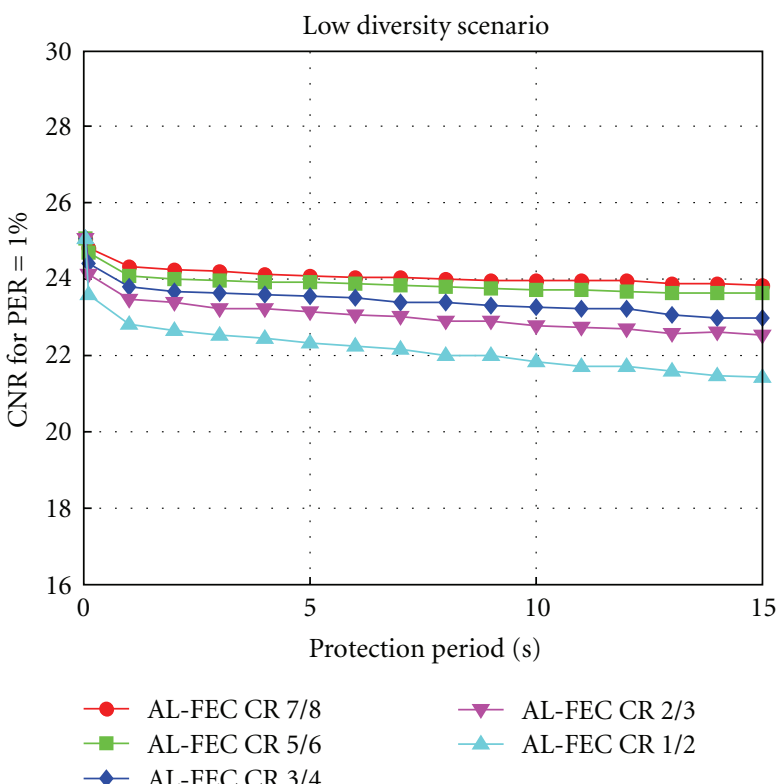

(a)

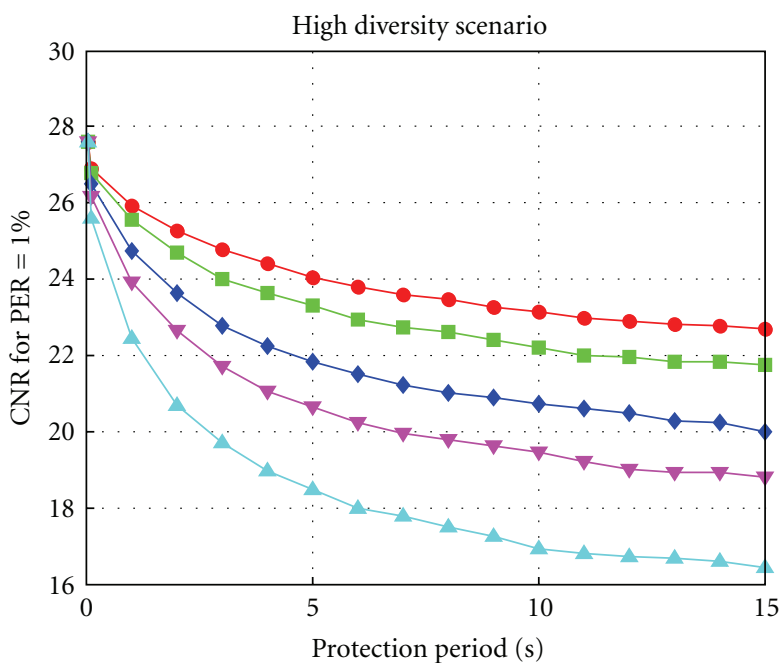

$\begin{aligned} \longrightarrow & \text { AL-FEC CR 7/8 } \\ - & \text { AL-FEC CR 5/6 } \\ - & \text { AL-FEC CR 3/4 }\end{aligned}$

$\rightarrow$ AL-FEC CR $2 / 3$

$\rightarrow$ AL-FEC CR $1 / 2$

(b)

Figure 6: Performance obtained by AL-FEC (a) in the low diversity scenario (correlation distance of $100 \mathrm{~m}$ and Doppler of $10 \mathrm{~Hz}$ ) and (b) in the high diversity scenario (correlation distance of $20 \mathrm{~m}$ and Doppler of $80 \mathrm{~Hz}$ ).

to the larger source packet size. In the figure we can also observe the little impact that the protection period has in a TU6 channel with high Doppler. Almost all the values achieve the same performance, and 0.1 seconds of protection period looses less than $1 \mathrm{~dB}$ with respect to 10 seconds. As it has been demonstrated, longer protection periods than 1 second do not provide a remarkable advantage in a TU6 channel.
5.2. Mobile Performance in the Presence of Fast Fading and Shadowing. In Figure 6 we can see the evolution of the performance of AL-FEC as the protection period increases for the two scenarios with shadowing defined for the simulations. We can observe an increase in the requirements of CNR caused by the effect of shadowing in mobile reception.

MPE-FEC performance is not presented as the results are approximately the same as with an AL-FEC configuration 
of 0.1 seconds. The results show the little impact of the protection period in low diversity conditions. In the low diversity scenario, there is almost no gain when increasing the protection period, especially in the case of the less robust code rates. Increasing the protection period from 0.1 to 15 seconds only yields a gain between 1 and $2 \mathrm{~dB}$ depending on the code rate. On the contrary, increasing the protection period from 0.1 to 15 seconds provides a gain from 4 to $10 \mathrm{~dB}$ in the high diversity scenario.

\section{Implementation Issues of AL-FEC}

6.1. FEC Implementation Issues. In this paper we have evaluated so far the performance of an ideal AL-FEC implementation in DVB-T. In a practical implementation some additional issues need to be addressed. A main issue is the transmission of the additional information required in reception for the decoding process. As already mentioned, we apply a scheme using hash sequences along with the repair symbols. The hash sequences represent at most a $10 \%$ of the total FEC data, so some very minor additional overhead must be taken into account in the configuration of the AL-FEC mechanism when compared to the results from above.

Another issue is the practical size of the source packets. Depending on the limitations of the hash mechanism employed, mainly the maximum number of source and repair packets per source block, the size of the source packets may have to be larger than 184 bytes. In this case, the loss of a single MPEG-2 TS packet results in a drop of more information in the source block. The simulation results have shown how the presence of isolated erroneous TS packets is more common at high Doppler values whereas it is less problematic at the low and medium range of Doppler.

An AL-FEC prototype for the protection of DVB-T services has been developed as a result of the collaboration between the Universidad Politécnica de Valencia and Digital Fountain. The prototype employs Raptor codes integrated into an IPTV streaming framework and operates with a source packet size of 1288 bytes and a repair packet size of 1472 bytes that corresponds exactly to a payload of 7 and 8 MPEG-2 TS packets, respectively. Figure 7 shows the performance obtained by the prototype. There is a degradation of $2 \mathrm{~dB}$ for the higher values of Doppler and less than $1 \mathrm{~dB}$ for the low and medium range of Doppler with respect to the results obtained in Figure 4. Note that this implementation is not yet fully optimized, but it shows the feasibility of the approach in a practical environment by reusing an IPTV streaming framework in the context. It is expected that future optimizations in the hash mechanism will allow AL-FEC to operate with smaller source and repair packets. Improved hash mechanisms specifically adapted to the integration in MPEG-2 TS packets are expected to move the results of real implementations even closer to the ideal performance shown in this paper.

The implementation of long protection periods is problematic in terms of channel switching time and memory requirements. As it has been explained, longer protection periods involve higher requirements of memory in the user

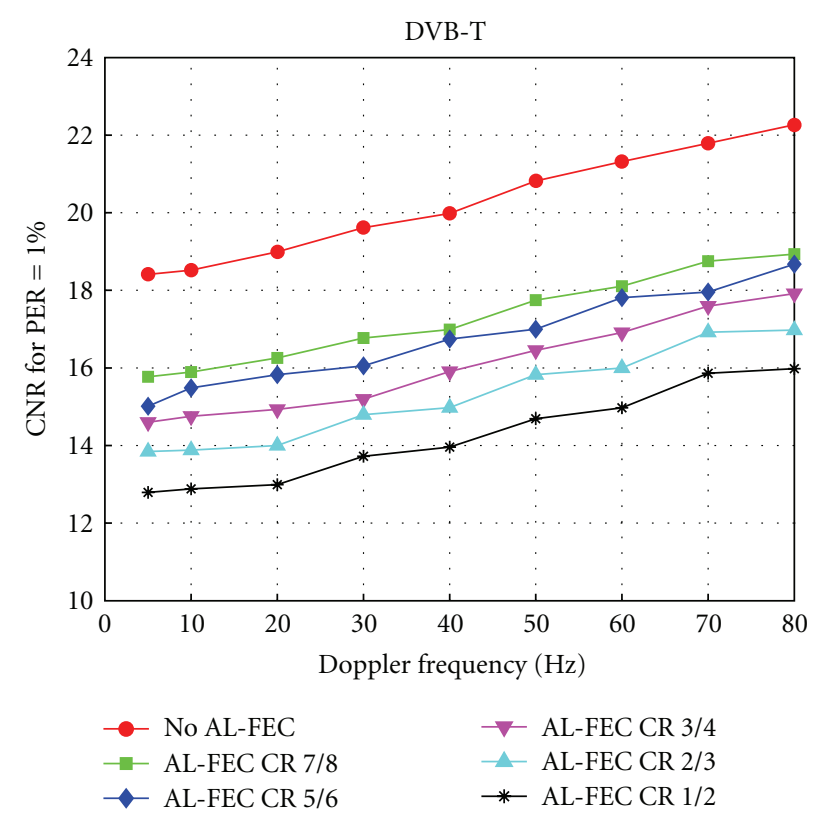

FIgUre 7: Mobile Performance of DVB-T services with a practical implementation of AL-FEC in a TU6 channel

terminals along with an increase in the channel switching time experimented by the user. Assuming a DVB-T typical service of $2.5 \mathrm{Mbps}$ and a protection period of 10 seconds, a minimum memory capacity of $3 \mathrm{MB}$ is required in order to store all the information of one source block. On the other hand, the use of computationally efficient erasure codes like Raptor avoids the implementation of dedicated hardware and allows the incorporation of AL-FEC as a software update.

Longer protection periods also have an important impact in channel switching time. Long channel switching times can degrade the user experience, and must be taken into consideration. Values of less than 0.5 seconds are not perceived by the user whereas values up to 2 seconds are considered to be tolerable [11]. As we have seen in the simulations, values of 1 second are capable to cope with fast fading and can even bring important gains in high diversity scenarios. Fast channel switching techniques are a hot topic today among mobile TV research as they can reduce the channel switching time perceived by the user with a small impact in the protection period. Although they are generally applied to bursty transmissions like DVB-H, they may also be incorporated in DVB-T services in order to improve the user experience when using long protection periods.

6.2. Deployment in Existing Systems. The available bandwidth for FEC data in DVB-T transmissions is also an important issue for the utilization of AL-FEC in existing DVB-T systems. The maximum amount of FEC data that can be multiplexed in an MPEG-2 TS depends on the available bandwidth and is limited. Existing DVB-T networks were not planned with mobile reception in mind and no specific bandwidth is assigned for the carriage of FEC data. Despite of this, it is possible to multiplex a limited amount 
of FEC data by means of the null TS packets generally present in an MPEG-2 TS. DVB-T transmitters insert null TS packets filled with stuffing data in the MPEG-2 TS in order to maintain a constant bit rate at the physical layer. We have performed preliminary studies in the MPEG-2 TS used in actual German and Spanish DVB-T transmissions to estimate the amount of null TS packets. The results reveal that depending on the multiplex, a percentage between $2 \%$ and $11 \%$ of the MPEG-2 TS corresponds to null TS packets that can be replaced by TS packets carrying FEC data.

DTT networks generally transmit several TV programs per MPEG-2 TS. Because of the limitation in the amount of available bandwidth to accommodate the FEC data, it may be desired to protect only few of the programs transmitted in an MPEG-2 TS. It is also possible to encode only a set of the video frames in order to increase the efficiency of the FEC data. Mobile reception in handheld terminals is generally performed with small displays that do not require the high frame rates of DVB-T services. The protection of Intra (I) and Predicted (P) frames only can increase the efficiency of the FEC data in a 33\% without major penalties in the user experience ( $B$ frames usually represent $1 / 3$ of the total amount of video frames). The arrangement of the video frames in each protection period can also affect the performance of the AL-FEC mechanism. Video frames in MPEG-2 video are grouped in Group of Pictures (GoP). By encoding an entire number of GoPs in each protection period, the errors do not propagate between protection periods, increasing the user experience for the same amount of uncorrected errors. The use of new video encoding standards such as H.264/AVC instead of the legacy MPEG-2 video coding, may also provide additional spare bandwidth for the accommodation of FEC data. By means of emerging video encoders and coding standards, it is possible to reduce the bit rate of current services without degrading the quality of the video experienced by the user.

\section{Network Planning Discussion}

Our results have shown that the AL-FEC protection of DVB$\mathrm{T}$ services is capable to obtain gains of $6 \mathrm{~dB}$ in mobile channels. However, other considerations apart from the fast fading must be taken into account in the planning of DVB-T networks for the provision of mobile TV.

While fixed reception is generally performed with high gain antennas located in the roof of the buildings, mobile reception is characterized by the reception at ground level and the use of low gain antennas. The added penalization of the height loss and the use of mobile antennas represent more than $20 \mathrm{~dB}$ if external antennas are used and more than $27 \mathrm{~dB}$ if integrated antennas are used instead [12]. This degradation has an important impact on the link budget of DVB-T systems, especially in urban scenarios. Therefore, the provision of mobile TV in DVB-T networks planned for fixed reception cannot be performed with coverage levels comparable to those of fixed DTT services. In this kind of networks, the AL-FEC protection of DVB-T services or the use of antenna diversity techniques can only be aimed to the provision of a best effort kind of service available in areas with the best coverage conditions.

On the other hand, DVB-T networks planned for portable reception like in Germany, take into account the penalization due to the height loss and lower gain antennas. However, in order to provide the necessary bit rate for DVB$\mathrm{T}$ services, DVB-T networks normally operate with higher modulation orders and less robust physical layer code rates than mobile TV networks such as DVB-H. Additionally, portable reception in DVB-T networks is planned for a Rayleigh channel model, which is approximately between 5 and $10 \mathrm{~dB}$ less demanding than mobile channels like the TU6 channel model. The combined gain of AL-FEC and antenna diversity techniques can be used in DVB-T networks deployed for portable reception in order to provide mobile DVB-T services with a similar coverage area to that of fixed DVB-T services.

\section{Conclusions}

In this paper we have investigated the use of AL-FEC for the provision of mobile DVB-T services. AL-FEC can be implemented in a backward compatible way and can be used in existing networks and services to extend the mobile coverage of DVB-T services. AL-FEC protection can be used in conjunction with antenna diversity techniques and hierarchical modulations in order to further enhance the vehicular reception of DVB-T services in existing networks. At the same time, newly deployed networks can be planned for the simultaneously provision of fixed and mobile DVB-T services.

The protection provided by AL-FEC depends not only on the proportion of FEC data transmitted along with the service information but also on the protection period. Long protection periods take advantage of the temporal diversity derived from user mobility, and achieve a better protection in the presence of shadowing.

We have provided direct comparisons between the ALFEC and MPE-FEC protection of DVB-T and DVB-H services in a TU6 channel. The results show that AL-FEC achieves an additional $4 \mathrm{~dB}$ gain with respect to MPE-FEC in the low range of Doppler. The additional gain at low Doppler values is motivated by the fact that AL-FEC can be configured with higher protection periods than MPE-FEC while having a little impact in the channel switching time. Long protection periods have been also investigated in TU6 channels affected by shadowing. The results show that the gain derived from long protection periods is heavily conditioned to the temporal diversity. A protection period of 10 seconds can provide a gain of $10 \mathrm{~dB}$ in high diversity scenarios. The use of longer protection periods involves higher memory requirements along with an increment in the channel switching time. The use of memory efficient decoding algorithms can solve the memory problems whereas fast channel switching techniques may decrease the channel switching time perceived by the user.

The implementation of AL-FEC in DVB-T presents a series of practical issues. The size of the source packets 
and the amount of additional information that must be transmitted have an important impact in the performance of the system. Current implementations of AL-FEC in DVB-T can loss about $2 \mathrm{~dB}$ with respect to the ideal implementation, especially at high velocities. The amount of bandwidth available in current DVB-T transmissions in order to accommodate FEC data is also an important issue. Several possibilities such as the protection of only a few services per MPEG-2 TS have been discussed in order to increase the efficiency of the transmitted FEC data.

\section{References}

[1] ETSI, EN 300744 v1.5.1, "Framing structure, channel coding and modulation for digital terrestrial television," November 2004.

[2] G. Faria, J. A. Henriksson, E. Stare, and P. Talmola, "DVB-H: digital broadcast services to handheld devices," Proceedings of the IEEE, vol. 94, no. 1, pp. 194-209, 2006.

[3] "Celtic WingTV Project: Services to Wireless, Integrated, Nomadic, GPRS-UMTS \& TV Handheld Terminals, WingTV Simulation Re-port, Deliverable 15b," December 2006.

[4] D. Gómez-Barquero, D. Gozálvez, and N. Cardona, "Application layer FEC for mobile TV delivery in IP datacast over DVB$\mathrm{H}$ systems," IEEE Transactions on Broadcasting, vol. 55, no. 2, pp. 396-406, 2009.

[5] "Advantages of Diversity Implementations on Mobile and Portable TV Receivers," Dibcom Whitepaper, May 2008.

[6] A. Shokrollahi, "Raptor codes," IEEE Transactions on Information Theory, vol. 52, no. 6, pp. 2251-2567, 2006.

[7] S. Saunders, Antennas and Propagation for Wireless Communications, John Wiley \& Sons, New York, NY, USA, 2000.

[8] ETSI, DVB Bluebook A131, “MPE-iFEC,” November 2008.

[9] ETSI, DVB Bluebook A054r4.1, "Interaction channel for Satellite Distribution Systems,” January 2009.

[10] ITU-T Recommendation H.222.0, "Generic coding of moving pictures and associated audio information: systems," April 2006.

[11] H. Fuchs and N. Farber, "Optimizing channel change time in IPTV applications," in Proceedings of IEEE International Symposium on Broadband Multimedia Systems and Broadcasting (BMSB '08), pp. 1-8, Las Vegas, Nev, USA, 2008.

[12] ETSI, "Implementation guidelines for DVB ter-restrial services; transmission aspects," Tech. Rep. TR 101190 v1.3.1, ETSI, Sophia Antipolis, France, November 2008. 

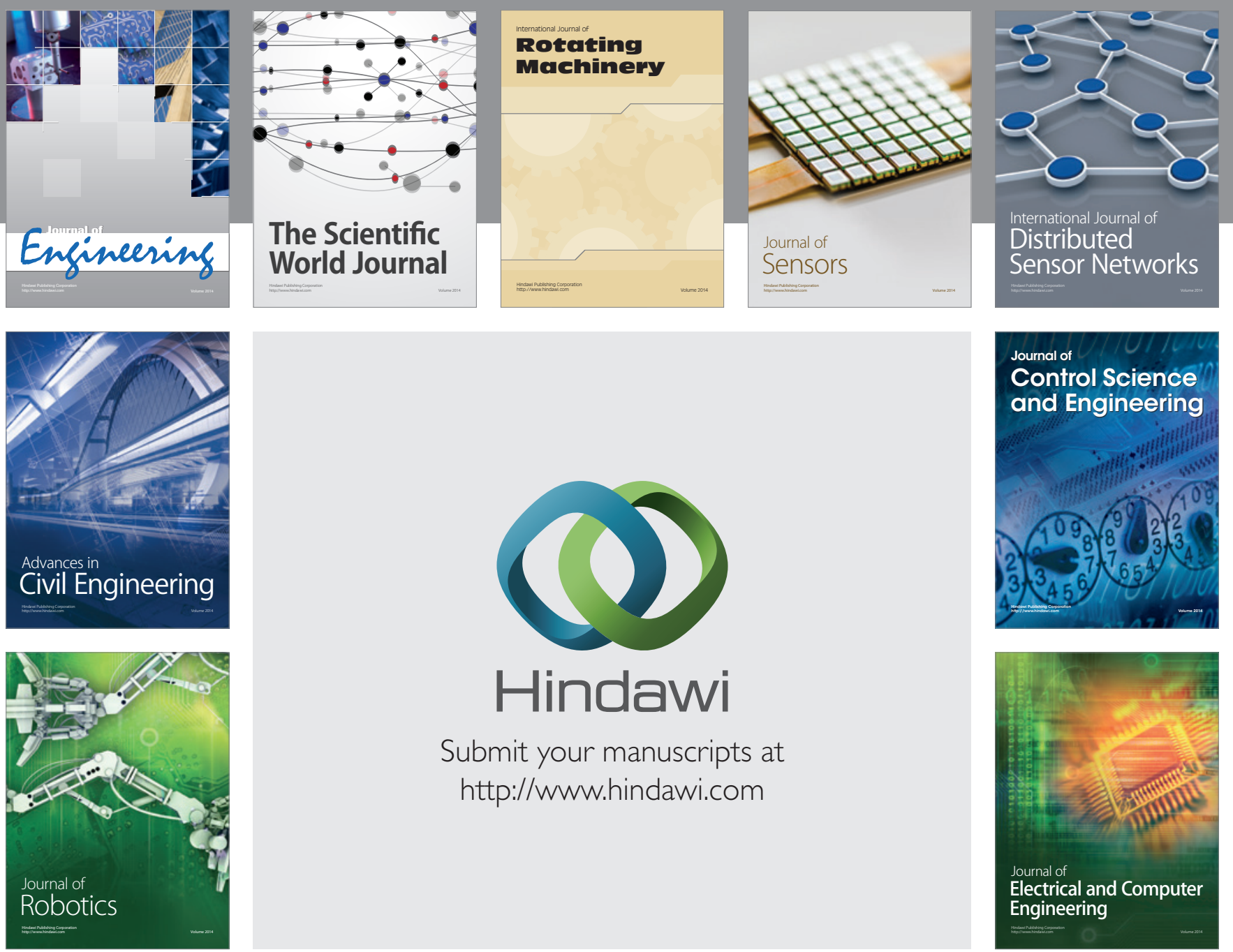

Submit your manuscripts at

http://www.hindawi.com
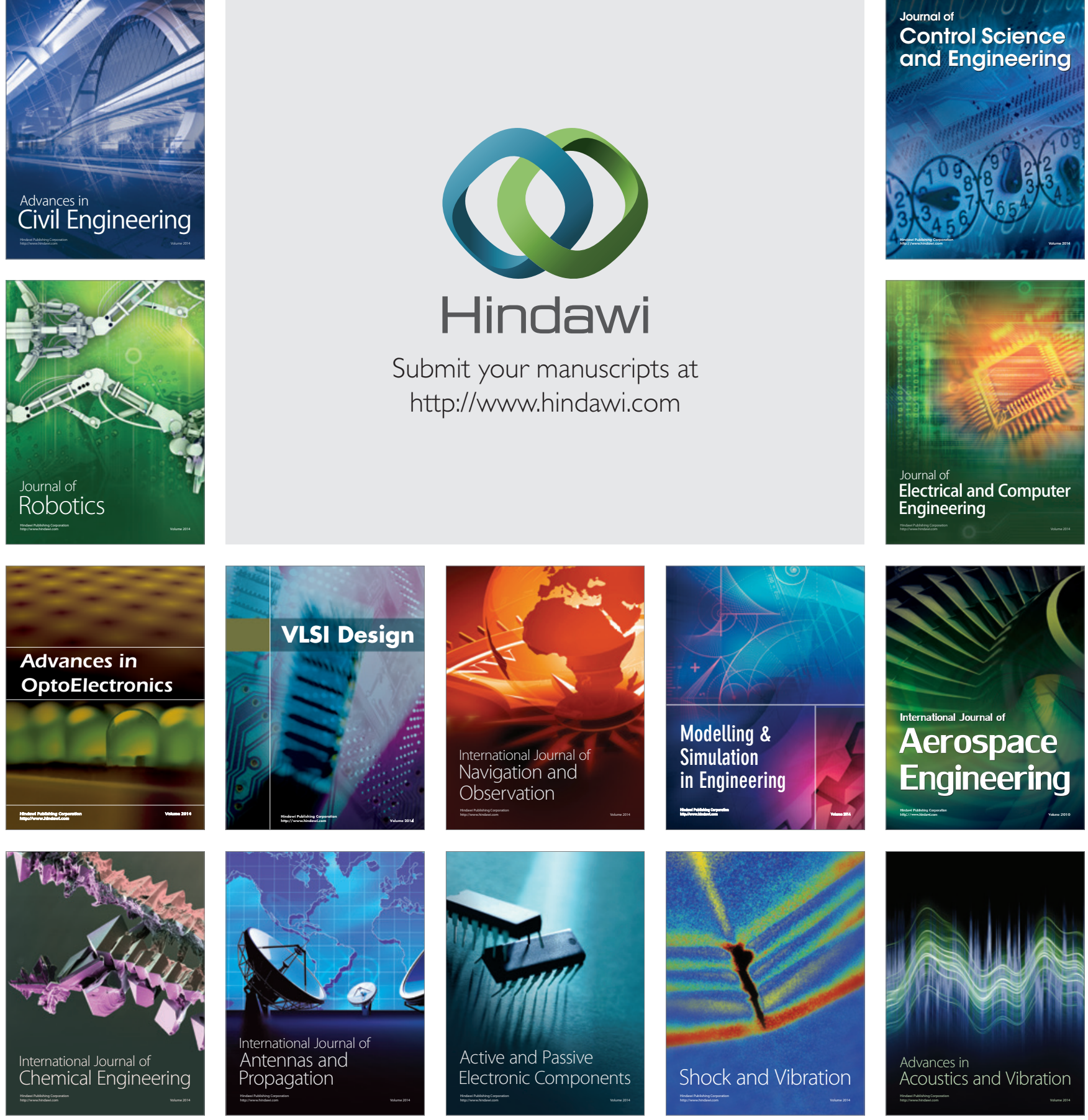